\title{
Гальваномагнитные свойства в анизотропных слоистых пленках на основе халькогенидов висмута
}

\author{
(C) О.А. УСов, Л.Н. Лукьянова, М.П. Волков
}

Физико-технический институт им. А.Ф. Иофрфе Российской академии наук, 194021 Санкт-Петербург, Россия

E-mail: oleg.usov@mail.ioffe.ru

Поступила в Редакцию 12 августа 2021 г.

В окончательной редакции 28 августа 2021 г.

Принята к публикации 28 августа 2021 г.

\begin{abstract}
В анизотропных слоистых пленках многокомпонентного твердого раствора $n$ - $\mathrm{Bi}_{1.92} \mathrm{In}_{0.02} \mathrm{Te}_{2.85} \mathrm{Se}_{0.15}$ в сильных магнитных полях от 2 до 14 Тл при низких температурах проведено исследование квантовых осцилляций магнетосопротивления, связанных с поверхностными состояниями электронов в 3D топологических изоляторах. Из анализа спектрального распределения амплитуд квантовых осцилляций магнетосопротивления определены основные параметры поверхностных состояний фермионов Дирака. Проведено сравнение результатов с данными, полученными методом сканирующей туннельной спектроскопии. Показано, что высокая поверхностная концентрация определяет вклад поверхностных состояний фермионов Дирака в термоэлектрические свойства пленок $n-\mathrm{Bi}_{1.92} \mathrm{In}_{0.02} \mathrm{Te}_{2.85} \mathrm{Se}_{0.15}$.
\end{abstract}

Ключевые слова: теллурид висмута, пленки, твердые растворы, топологический изолятор, осцилляции магнетосопротивления.

DOI: 10.21883/FTP.2021.12.51692.08

\section{1. Введение}

Возможность практического использования топологических явлений, обнаруженных в большинстве термоэлектриков, среди которых твердые растворы на основе халькогенидов висмута являются одними из перспективных материалов [1], определяет необходимость исследования аномальных свойств топологических поверхностных состояний фермионов Дирака с линейной дисперсией и сильной связью между спином и импульсом, препятствующей рассеянию фермионов на немагнитных примесях и дефектах [2]. В настоящее время свойства поверхностных электронных состояний, обнаруженные в $\mathrm{Bi}_{2} \mathrm{Te}_{3}$, применяются в фотонике в системах оптической связи для синхронизации оптоволоконных лазеров $[3,4]$. В термоэлектричестве и электронике активно исследуется эффект сверхтекучести экситонов [5,6], приводящий к увеличению проводимости.

Новый подход использования топологических поверхностных состояний связан со спин-эффектом Зеебека, который наблюдается в гетероструктурах, состоящих из топологического изолятора $\left(\mathrm{Bi}_{x} \mathrm{Sb}_{1-x}\right)_{2} \mathrm{Te}_{3}$ и ферримагнитного изолятора иттриевого феррита-граната (YIG) [7]. В пленке YIG при наличии градиента температуры генерируются неравновесные спиновые волны (магноны), а в топологическом изоляторе $\left(\mathrm{Bi}_{x} \mathrm{Sb}_{1-x}\right)_{2} \mathrm{Te}_{3}$ при оптимальном положении уровня Ферми создается электрическое напряжение за счет спинового эффекта Зеебека.

Практическое использование вклада топологических поверхностных состояний фермионов Дирака в термоэлектрические свойства было обнаружено в области электронных изоструктурных топологических фазовых переходов в слоистых пленках многокомпонентных термоэлектриков на основе теллурида висмута $[8,9]$ при давлениях 3-4ГПа, которым соответствует повышение параметра мощности в 2.5 раза при комнатной температуре по сравнению с нормальными условиями.

Детальная информация об особенностях топологических поверхностных состояний фермионов Дирака в термоэлектриках на основе халькогенидов висмута может быть получена из исследований гальваномагнитных свойств в сильных магнитных полях [10-12]. К основным параметрам, которые определяют из осцилляций магнетосопротивления при низких температурах, относятся: частота циклотронного резонанса $F$, поверхностная концентрация фермионов $n_{s}$, волновой вектор $k_{\mathrm{F}}$, циклотронная эффективная масса $m_{\text {сус }}$, скорость Ферми $v_{\mathrm{F}}$, энергия Ферми $E_{\mathrm{F}}$, время релаксации $\tau$, длина свободного пробега $l_{\mathrm{F}}$ и подвижность $\mu$ фермионов.

Для исследования поверхностных электронных состояний фермионов Дирака [13] в последние годы используют метод сканирующей туннельной спектроскопии (СТC). Этот метод позволяют определить дифференциальную туннельную проводимость, пропорциональную электронной плотности состояний, положение точки Дирака и другие параметры, некоторые из которых получают также из осцилляционных эффектов.

Настоящая работа посвящена исследованию квантовых осцилляций магнетосопротивления в магнитных полях до $B=14$ Тл при низких температурах в анизотропных слоистых пленках многокомпонентного твердого раствора $n-\mathrm{Bi}_{1.92} \mathrm{In}_{0.02} \mathrm{Te}_{2.85} \mathrm{Se}_{0.15}$ с оптимальными термоэлектрическими свойствами вблизи комнатной температуры с целью определения параметров топологических поверхностных состояний фермионов Дирака. Проводится сравнение полученных результатов с данными 
исследований спектров дифференциальной туннельной проводимости, полученных методом СТС.

\section{2. Квантовые осцилляции магнетосопротивления. Частота осцилляций магнетосопротивления}

Квантовые осцилляции магнетосопротивления, возникающие в кристаллических материалах в магнитном поле в результате периодической модуляции плотности состояний электронов с частотой циклотронного резонанса $F$, описываются как функция магнитного поля $B$ в модели Лифшица-Косевича [14] и используются для анализа и определения параметров поверхностных состояний фермионов Дирака в топологических изолятоpax [15].

Исследования топологических поверхностных состояний фермионов Дирака в анизотропных слоистых пленках многокомпонентных твердых растворов $n$ - $\mathrm{Bi}_{1.92} \mathrm{In}_{0.02} \mathrm{Te}_{2.85} \mathrm{Se}_{0.15}$ проводились на основе анализа зависимостей поперечного магнетосопротивления $\rho_{x x}$, измеренного на установке Physical Property Measurement System (PPMS) Transport Option в магнитных полях до $B=14$ Тл при низких температурах $T=3,5,10 \mathrm{~K}$. Пленки от субмикронной толщины до 300 нм были получены с помощью липкой ленты последовательным расслоением монокристаллических зерен объемных твердых растворов вдоль межслоевой ван-дер-ваальсовой поверхности (0001), которая перпендикулярна кристаллографической оси 3-го порядка $c$.

Экспериментальные зависимости магнетосопротивления от магнитного поля $\rho_{x x}(B)$ (рис. 1) после сглаживания для удаления шумов и вычитания фоновых линий, аппроксимированных полиномами третьей степени, использовали для построения осцилляций магнетосопротивления $\Delta \rho_{x x}$ от обратной величины магнитного



Рис. 1. Зависимости магнетосопротивления $\Delta \rho_{x x}(1-3)$ от магнитного поля $B$ в пленке $n-\mathrm{Bi}_{1.92} \mathrm{In}_{0.02} \mathrm{Te}_{2.85} \mathrm{Se}_{0.15}$ для температур $T, \mathrm{~K}: 1-3,2-5,3-10$.

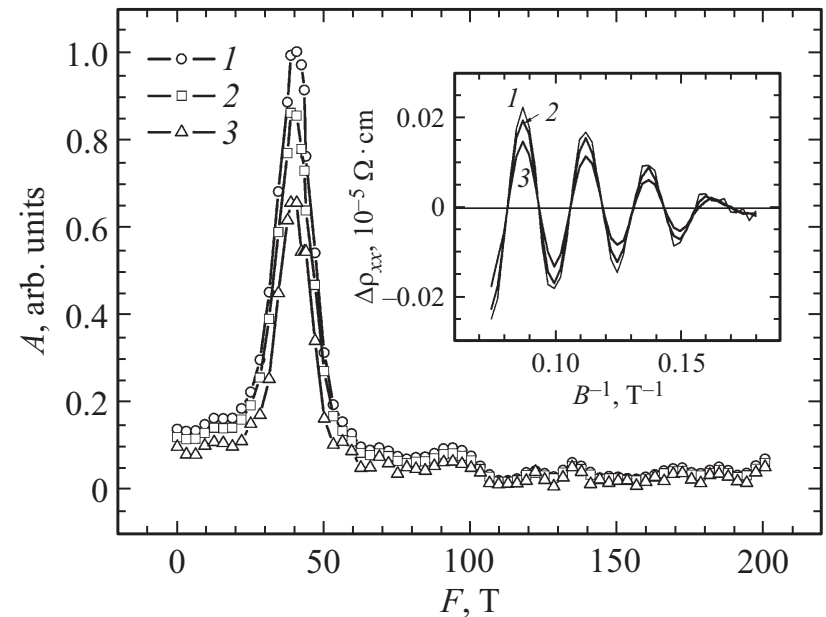

Рис. 2. Спектральные распределения амплитуд осцилляций магнетосопротивления $A$ с максимумом на частоте $F$ в пленке $n$ - $\mathrm{Bi}_{1.92} \mathrm{In}_{0.02} \mathrm{Te}_{2.85} \mathrm{Se}_{0.15}$, полученные после вычитания фона методом БПФ из зависимостей $\Delta \rho_{x x}\left(B^{-1}\right)$, приведенных на вставке при температурах 3,5 и $10 \mathrm{~K}$.

поля $B^{-1}$. Методом быстрого преобразования Фурье (БПФ) была определена частота циклотронного резонанса $F$ (рис. 2) из $\Delta \rho_{x x}\left(B^{-1}\right)$.

Из анализа спектрального распределения амплитуд осцилляций магнетосопротивления (рис. 2, кривые $1-3$ ) в пленке $n-\mathrm{Bi}_{1.92} \mathrm{In}_{0.02} \mathrm{Te}_{2.85} \mathrm{Se}_{0.15}$ следует, что циклотронная резонансная частота осцилляций $F=40$ Тл. В многокомпонентных твердых растворах на основе $\mathrm{Bi}_{2} \mathrm{Te}_{3}[11,16,17]$ возможно появление дополнительных резонансных частот вследствие искажений конуса Дирака, которые приводят к возникновению особенностей поверхности Ферми. Однако небольшие замещения атомов в обеих подрешетках $\mathrm{Bi}$ и Те в пленке $n$ - $\mathrm{Bi}_{1.92} \mathrm{In}_{0.02} \mathrm{Te}_{2.85} \mathrm{Se}_{0.15}$ не приводят к искажению спектрального распределения амплитуд осцилляций магнетосопротивления (рис. 2) по сравнению с твердым раствором $\mathrm{Bi}_{1.6} \mathrm{Sb}_{0.4} \mathrm{Te}_{2.94} \mathrm{Se}_{0.06}$ с большим суммарным количеством замещенных атомов [17]. Максимальные значения амплитуд осцилляций магнетосопротивления $A$ в зависимости от частоты $F$ уменьшаются с ростом температуры. Для описания сечения поверхности Ферми используется круг с центром в точке $\Gamma$ зоны Бриллюэна [15].

\section{3. Площадь сечения поверхности Ферми, волновой вектор, поверхностная концентрация фермионов}

В модели Лифшица-Косевича [14] были рассчитаны параметры поверхностных состояний фермионов Дирака для слоистых пленок $n$ - $\mathrm{Bi}_{1.92} \mathrm{In}_{0.02} \mathrm{Te}_{2.85} \mathrm{Se}_{0.15}$ из анализа спектрального распределения амплитуд квантовых осцилляций магнетосопротивления (рис. 2, кривые $1-3$ ). 
Частота циклотронного резонанса $F$, сечение поверхности Ферми $S\left(k_{\mathrm{F}}\right)$, волновой вектор $k_{\mathrm{F}}$, энергия Ферми $E_{\mathrm{F}}$, поверхностная концентрация фермионов $n_{s}$, циклотронная эффективная масса $m_{\text {сус }} / m_{e}$, время релаксации $\tau$, температура Дингла $T_{\mathrm{D}}$, длина свободного пробега $l_{\mathrm{F}}$, скорость Ферми $v_{\mathrm{F}}$, подвижность $\mu$, и циклотронная частота $w$ для пленки $\mathrm{Bi}_{1.92} \mathrm{In}_{0.02} \mathrm{Te}_{2.85} \mathrm{Se}_{0.15}$

\begin{tabular}{c|c|c|c|c|c|c|c|c|c|c|c}
\hline$F, \mathrm{~T}$ & $S\left(k_{\mathrm{F}}\right), \mathrm{HM}^{-2}$ & $k_{\mathrm{F}}, \mathrm{HM}^{-1}$ & $E_{\mathrm{F}}, \mathrm{M \ni B}$ & $n_{s}, 10^{12}, \mathrm{~cm}^{-2}$ & $m_{\mathrm{cyc}} / m_{e}$ & $\tau, 10^{-13}, \mathrm{c}$ & $T_{\mathrm{D}}, \mathrm{K}$ & $l_{\mathrm{F}}, \mathrm{HM}$ & $v_{\mathrm{F}}, 10^{5}, \mathrm{M} / \mathrm{c}$ & $\mu, \mathrm{M}^{2}(\mathrm{~B} \cdot \mathrm{c})$ & $w, \mathrm{~T} Г \mathrm{~L}$ \\
\hline 40 & 0.38 & 0.35 & 77.2 & 1.0 & 0.12 & 3.4 & 3.59 & 114 & 3.36 & 0.50 & 5.86 \\
\hline$[17] 10$ & & 0.17 & 6.43 & 0.24 & 0.18 & 2.9 & 4.2 & 32.1 & 1.12 & 0.25 \\
{$[17] 27$} & & 0.29 & 26.0 & 0.65 & 0.12 & 3.5 & 3.4 & 98 & 2.76 & 0.51
\end{tabular}

Согласно соотношениям Онзагера [14], частота квантовых осцилляций магнетосопротивления $F$ пропорциональна площади сечения поверхности Ферми $S\left(k_{\mathrm{F}}\right)$ :

$$
F=\left(\frac{\hbar}{2 \pi e}\right) S\left(k_{\mathrm{F}}\right),
$$

где $k_{\mathrm{F}}-$ волновой вектор электрона, для сечения поверхности Ферми $S\left(k_{\mathrm{F}}\right)$ в форме круга

$$
k_{\mathrm{F}}=\sqrt{\frac{S\left(k_{\mathrm{F}}\right)}{\pi}} .
$$

Поверхностная концентрация фермионов $n_{s}$ определяется в виде

$$
n_{s}=\frac{k_{\mathrm{F}}^{2}}{4 \pi} \text {. }
$$

Поверхностная концентрация фермионов $n_{s}$ является одним из параметров, определяющих вклад поверхностных состояний фермионов Дирака в термоэлектрические свойства материала. В пленке $n$-Bi ${ }_{1.92} \operatorname{In}_{0.02} \mathrm{Te}_{2.85} \mathrm{Se}_{0.15}$ с высоким параметром мощности, равным $46.3 \cdot 10^{-6} \mathrm{BT} \cdot \mathrm{cm}^{-1} \mathrm{~K}^{-2}$, величина $n_{s}=1 \cdot 10^{12} \mathrm{~cm}^{-2}$ при низких температурах в соответствии с $(1-3)$, была выше, чем в составе $\mathrm{Bi}_{1.6} \mathrm{Sb}_{0.4} \mathrm{Te}_{2.94} \mathrm{Se}_{0.06}$ [17] (см. таблицу).

Исследования в твердых растворов $n$ - $\mathrm{Bi}_{1.92} \mathrm{In}_{0.02} \mathrm{Te}_{2.85} \mathrm{Se}_{0.15}$ дифференциальной туннельной проводимости методом сканирующей туннельной спектроскопии при комнатной температуре показали, что поверхностная концентрация $n_{s}=2.8 \cdot 10^{12} \mathrm{~cm}^{-2}[18]$. Как следует из полученных в настоящей работе данных, величина $n_{s}$ возрастает с температурой. Кроме того, величина $n_{s}$ в $n$ - $\mathrm{Bi}_{1.92} \operatorname{In}_{0.02} \mathrm{Te}_{2.85} \mathrm{Se}_{0.15}$ выше, чем в составах системы твердых растворов $n-\mathrm{Bi}_{2} \mathrm{Te}_{3-y} \mathrm{Se}_{y}$ [18]. Таким образом, увеличение вклада поверхностных состояний в пленке $n$ - $\mathrm{Bi}_{1.92} \mathrm{In}_{0.02} \mathrm{Te}_{2.85} \mathrm{Se}_{0.15}$ по сравнению с исследованными термоэлектриками определяется ростом поверхностной концентрации фермионов Дирака.

\section{4. Уровни Ландау}

Осцилляции магнетосопротивления в сильном магнитном поле связаны с изменениями заселенности уровней Ландау, энергия между которыми равна энергии циклотронного резонанса. Характер распределения заселенности описывается диаграммой уровней Ландау, в которой величины обратного магнитного поля, соответствующие минимумам амплитуд осцилляций, линейно зависят от целых индексов $n$, а максимумы - от по-

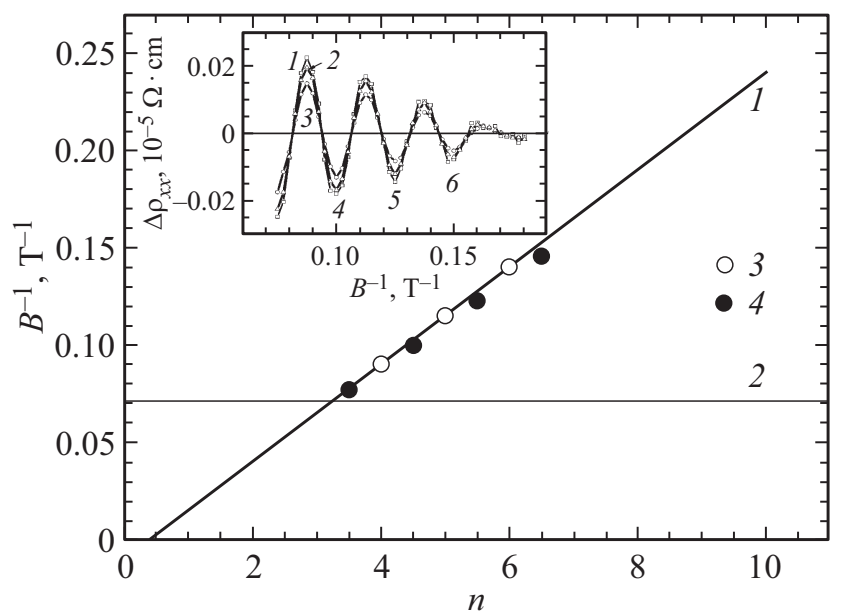

Рис. 3. Зависимость индексов уровней Ландау $n$ от обратного магнитного поля $B^{-1}$, в пленке $n$ - $\mathrm{Bi}_{1.92} \operatorname{In}_{0.02} \mathrm{Te}_{2.85} \mathrm{Se}_{0.15}$, полученная из минимумов амплитуд осцилляций $\Delta \rho_{x x}\left(B^{-1}\right)$ (вставка) при температурах $T, \mathrm{~K}: 1-3,2-5,3-10$. $n:$ 4, 5, 6 - индексы уровней Ландау. Точки 3 - минимумы, точки $4-$ максимумы амплитуд осцилляций $\Delta \rho_{x x}\left(B^{-1}\right)$. Линия 1 - линейная экстраполяция значений $n$, линия $2-$ соответствует максимальному значению магнитного поля $B=14$ Тл. Отклонение величины экспериментального фазового сдвига, связанного с фазой Берри, являющейся характеристикой кривизны циклотронной орбиты и дисперсии электронов [19], может быть обусловлено влиянием зеемановского расщепления и искажением линейной дисперсии дираковских фермионов.

луцелых индексов уровней Ландау [14]. Линейная зависимость, построенная методом наименьших квадратов (МНК) на периодической совокупности минимумов и максимумов амплитуд осцилляций магнетосопротивления в пленке $n$ - $\mathrm{Bi}_{1.92} \operatorname{In}_{0.02} \mathrm{Te}_{2.85} \mathrm{Se}_{0.15}$, позволяет определить индексы уровней Ландау $n$, которые равны 4, 5,6 (рис. 3). Продолжение линейной зависимости (рис. 3, кривая 1) до пересечения с осью индексов уровней Ландау $n$ позволяет определить сдвиг, соответствующий фазе Берри при $\beta=0.4$.

\section{5. Циклотронная эффективная масса}

Циклотронная эффективная масса $m_{\text {сус }}$ была определена методом МНК из температурной зависимости ампли- 


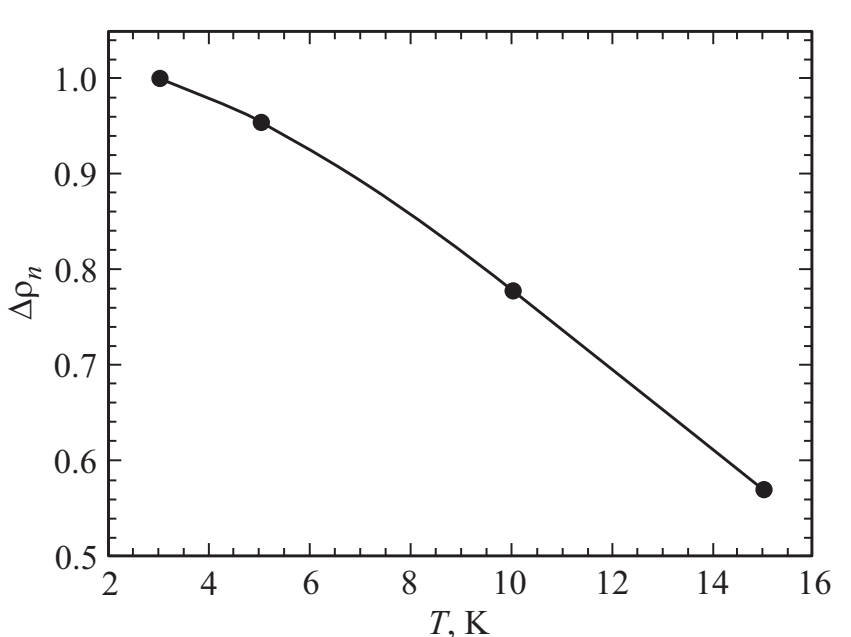

Рис. 4. Температурная зависимость амплитуд осцилляций магнетосопротивления $\Delta \rho_{n}\left(\right.$ Тл, $\left.B^{-1}\right)$ при максимальных значениях амплитуд в магнитном поле $B=13.3$ Тл, нормированных на амплитуду при $T=3 \mathrm{~K}$, в пленке $n$ - $\mathrm{Bi}_{1.92} \mathrm{In}_{0.02} \mathrm{Te}_{2.85} \mathrm{Se}_{0.15}$.



Рис. 5. Зависимость функции Дингла $\ln \left(\Delta \rho_{n}(T / B) \sinh (\alpha T / B) /(\alpha T / B)\right)$ от обратного магнитного поля $B^{-1}$, определяющая температуру Дингла $T_{\mathrm{D}}$ в пленке $n-\mathrm{Bi}_{1.92} \mathrm{In}_{0.02} \mathrm{Te}_{2.85} \mathrm{Se}_{0.15}$, для которой $T_{\mathrm{D}}=3.6 \mathrm{~K}$.

туд осцилляций магнетосопротивления при максимальных значениях амплитуд в магнитном поле $B=13.3$ Тл


осцилляций магнетосопротивления были нормированы к амплитуде, соответствующей минимальной температуре $3 \mathrm{~K}$ (рис. 4). Функция температурной зависимости амплитуд осцилляций магнетосопротивления имеет вид: $\Delta \rho_{n}\left(m_{c} T B^{-1}\right)=\left(\alpha_{0} m_{c} T B^{-1}\right) / \sinh \left(\alpha_{0} m_{c} T B^{-1}\right)$, где $\alpha_{0}=2 \pi^{2} k_{\mathrm{B}} / \hbar m_{e} / e=14.69 \mathrm{~T} \pi \cdot \mathrm{K}^{-1}, m_{c}=m_{\mathrm{cyc}} / m_{e}$. $k_{\mathrm{B}}, \hbar, m_{e}, e-$ константа Больцмана, приведенная константа Планка, масса и заряд электрона соответственно. Полученная величина $m_{\text {сус }}=0.12$ вместе с волновым вектором $k_{\mathrm{F}}$ позволяют определить скорость $v_{\mathrm{F}}$ и энергию Ферми $E_{\mathrm{F}}$ (см. таблицу).
По данным СТС [18], в пленке $n$ - $\mathrm{Bi}_{1.92} \operatorname{In}_{0.02} \mathrm{Te}_{2.85} \mathrm{Se}_{0.15}$ точка Дирака $E_{\mathrm{D}}$, положение которой существенно зависит от состава материала, расположена приблизительно в центре запрещенной зоны и на расстоянии 0.8 мэВ от края валентной зоны. Поэтому даже при температуре $3 \mathrm{~K}$ и энергии $E_{\mathrm{F}}=77$ мэВ уровень Ферми в пленке находится у края зоны проводимости. Это позволяет объяснить большое время релаксации Дингла $\tau$ и подвижности $\mu$, так как установлено [10], что в 3D топологических изоляторах $n$ - и $p$-типа в результате интерференции фермионов Дирака и объемных электронов зоны проводимости происходит сильное ослабление рассеяния фермионов, если уровень Ферми оказывается вблизи одного из краев запрещенной зоны.

\section{6. Температура Дингла}

Температура Дингла $T_{\mathrm{D}}$ и время релаксации рассеяния Дингла $\tau$ в модели Лифшица-Косевича описывают спад амплитуд осцилляций с уменьшением магнитного поля, связанного с рассеянием фермионов на примесях. Температура и время релаксации Дингла определяются из зависимости амплитуд осцилляции от магнитного поля при фиксированной температуре. В данном случае используются 4 максимума амплитуд осцилляций $\left(B^{-1}=0.1,0.125,0.15,0.175 \mathrm{~T}^{-1}\right)$ при температуре $T=3 \mathrm{~K}$ (рис. 5$)$.

Температура Дингла $T_{\mathrm{D}}$ была определена методом МНК из наклона линейной зависимости (рис. 5), которая описывается функцией $\ln \left(\Delta \rho_{n}(T / B) \sinh (\alpha T / B) /(\alpha T / B)\right)=\alpha T_{\mathrm{D}} / B \quad$ от обратного магнитного поля $B$. Константа $\alpha=1.763 \mathrm{Tл} \cdot \mathrm{K}^{-1}$ для пленки $n-\mathrm{Bi}_{1.92} \operatorname{In}_{0.02} \mathrm{Te}_{2.85} \mathrm{Se}_{0.15}$, так как зависит от циклотронной эффективной

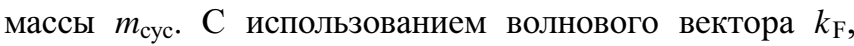
скорости Ферми $v_{\mathrm{F}}$ и эффективной массы $m_{\text {сус }}$ были рассчитаны следующие параметры фермионов Дирака: время релаксации $\tau$, длина свободного пробега $l_{\mathrm{F}}$ и подвижность электронов $\mu$, представленные в таблице.

Длина свободного пробега фермионов $l_{\mathrm{F}}=114 \mathrm{нм} \mathrm{в}$ пленке $n$ - $\mathrm{Bi}_{1.92} \mathrm{In}_{0.02} \mathrm{Te}_{2.85} \mathrm{Se}_{0.15}$ существенно выше, чем в твердом растворе $\mathrm{Bi}_{1.6} \mathrm{Sb}_{0.4} \mathrm{Te}_{2.94} \mathrm{Se}_{0.06}$ (см. таблицу). Значение $l_{\mathrm{F}}$ обычно заметно меньше длины квантовой фазовой когерентности $l_{\varphi}$, связанной с процессами неупругого рассеяния электронов [12]. По оценкам в пленке $\mathrm{Bi}_{2} \mathrm{Te}_{3} l_{\varphi}=350$ нм при $4.2 \mathrm{~K}$, а длина свободного пробега $l_{\mathrm{F}} \sim 60 \mathrm{Hм}[20,21]$. При таких величинах $l_{\varphi}$ квантовые эффекты, связанные с поверхностными состояниями дираковских фермионов, могут наблюдаться не только в сверхтонких образцах, но и в образцах до нескольких сотен нанометров.

\section{7. Заключение}

В анизотропных слоистых пленках многокомпонентного твердого раствора $n$ - $\mathrm{Bi}_{1.92} \mathrm{In}_{0.02} \mathrm{Te}_{2.85} \mathrm{Se}_{0.15}$ с высо- 
ким параметром мощности, относящегося к топологическим изоляторам, исследованы квантовые осцилляции магнетосопротивления в сильных магнитных полях до $B=14$ Тл при низких температурах $3-10 \mathrm{~K}$. Параметры поверхностных состояний фермионов Дирака были рассчитаны в модели Лифшица-Косевича из анализа спектрального распределения амплитуд квантовых осцилляций магнетосопротивления. Идентифицированы экстремальные точки амплитуд осцилляций магнетосопротивления, определены соответствующие им индексы уровней Ландау и фаза Берри, подтверждающая топологический характер поверхностных состояний фермионов Дирака.

Сравнение величины энергии уровня Ферми $E_{\mathrm{F}}$ и поверхностной концентрации фермионов $n_{s}$ в пленке $n-\mathrm{Bi}_{1.92} \operatorname{In}_{0.02} \mathrm{Te}_{2.85} \mathrm{Se}_{0.15}$ с аналогичными данными, полученными при исследовании спектров дифференциальной туннельной проводимости методом сканирующей туннельной спектроскопии при комнатной температуре, показали, что величина $n_{s}$ возрастает с ростом температуры, а уровень Ферми при низких температурах находится вблизи края зоны проводимости.

Показано, что величина поверхностной концентрации фермионов $n_{s}$ в пленке $n$ - $\mathrm{Bi}_{1.92} \mathrm{In}_{0.02} \mathrm{Te}_{2.85} \mathrm{Se}_{0.15}$ при низких температурах до $1 \cdot 10^{12} \mathrm{~cm}^{-2}$ практически в 2 раза больше, чем в составе $\mathrm{Bi}_{1.6} \mathrm{Sb}_{0.4} \mathrm{Te}_{2.94} \mathrm{Se}_{0.06}$, что определяет увеличение вклада поверхностных состояний в термоэлектрические свойства в исследованной пленке за счет роста $n_{s}$.

\section{Финансирование работы}

Работа выполнена при финансовой поддержке Российского фонда фундаментальных исследований (грант № 20-08-00464).

\section{Конфликт интересов}

Авторы заявляют, что у них нет конфликта интересов.

\section{Список литературы}

[1] J. Heremans, R. Cava, N. Samarth. Nature Rev. Mater., 2, 17049 (2017).

[2] N. Xu, Y. Xu, J. Zhu. npj Quant. Mater., 2, 51 (2017).

[3] G. Jiang, J. Yi, L. Miao, P. Tang, H. Huang, C. Zhao, S. Wen. Sci. Rep., 8, 2355 (2018).

[4] L. Zhang, J. Liu, J. Li, Z. Wang, Y. Wang, Y. Ge, W. Dong, Xu, H. Zhang, W. Zhang. Laser Photon. Rev., 14, 1900409 (2020).

[5] R.V. Gorbachev, A.K. Geim, M.I. Katsnelson, K.S. Novoselov, T. Tudorovskiy, I.V. Grigorieva, A.H. MacDonald, S.V. Morozov, K. Watanabe, T. Taniguchi, L.A. Ponomarenko. Nature Physics, 8, 896 (2012).

[6] Y. Hou, R. Wang, R. Xiao, L. McClintock, H.C. Travaglini, J.P. Francia, H. Fetsch, O. Erten, S.Y. Savrasov, B. Wang, A. Rossi, I. Vishik, E. Rotenberg, D. Yu. Nature Commun., 10, 5723 (2019).
[7] Z. Jiang, C.-Z. Chang, M.R. Masir, C. Tang, Y. Xu, J.S. Moodera, A.H. MacDonald, J. Shi. Nature Commun., 7, 11458 (2016).

[8] И.В. Коробейников, Н.В. Морозова, Л.Н. Лукьянова, О.А. Усов, С.В. Овсянников. ФТП, 53, 741 (2019).

[9] I.V. Korobeinikov, N.V. Morozova, L.N Lukyanova, O.A Usov, V.A. Kulbachinskii, V.V Shchennikov, S.V. Ovsyannikov. J. Phys. D: Appl. Phys., 51 (2), 025501 (2018).

[10] H. Liu, S. Liu, Y. Yi, H. He, J. Wang. 2D Mater., 2, 045002 (2015).

[11] С.И. Веденеев. УФН, 187, 411 (2017).

[12] L. Bao, L. He, N. Meyer, X. Kou, P. Zhang, Z. Chen, A.V. Fedorov, J. Zou, T.M. Riedemann, T.A. Lograsso, K.L. Wang, G. Tuttle1, F. Xiu. Sci.Rep. 2, 726 (2012).

[13] X. He, H. Li, L. Chen, K. Wu. Sci. Rep., 5, 8830 (2015).

[14] D. Shoenberg. Magnetic oscillations in metals. Ser. Monographs on physics (Cambridge University Press, Cambridge, 2009).

[15] Y. Ando. J. Phys. Soc. Jpn., 82, 102001 (2013).

[16] N.H. Tu, Y. Tanabe, Y. Satake, K.K. Huynh, P.H. Le, S.Yu. Matsushita, K. Tanigaki. Nano Lett., 17, 2354 (2017).

[17] Л.Н. Лукьянова, О.А. Усов, М.П. Волков. ФТП, 53, 626 (2019).

[18] L.N. Lukyanova, I.V. Makarenko, O.A. Usov. J. Phys.: Condens. Matter, 32, 465701 (2020).

[19] A.R. Wright, R.H. McKenzie. Phys. Rev. B, 87, 085411 (2013).

[20] Л.Н. Лукьянова, Ю.А. Бойков, В.А. Данилов, О.А. Усов, М.П. Волков, В.А. Кутасов. ФТТ, 56, 907 (2014).

[21] Л.Н. Лукьянова, Ю.А. Бойков, В.А. Данилов, О.А. Усов, М.П. Волков. ФТП, 51, 880 (2017).

\section{Galvanomagnetic properties in anisotropic layered films based on bismuth chalcogenides}

\section{O.A. Usov, L.N. Lukyanova, M.P. Volkov}

loffe Institute, 194021 St. Petersburg, Russia

\begin{abstract}
In anisotropic layered films of a multicomponent $n$ - $\mathrm{Bi}_{1.92} \mathrm{In}_{0.02} \mathrm{Te}_{2.85} \mathrm{Se}_{0.15}$ solid solution in strong magnetic fields from 2 to $14 \mathrm{~T}$ at low temperatures, quantum oscillations of magnetoresistance associated with surface states of electrons in $3 \mathrm{D}$ topological insulators were studied. From the analysis of the spectral distribution of the amplitudes of quantum oscillations of magnetoresistance, the main parameters of the Dirac fermion surface states are determined. A comparison of the results with the data obtained by the method of scanning tunnel spectroscopy was carried out. It is shown that a high surface concentration determines the contribution of the Dirac fermion surface states to the thermoelectric properties of $n-\mathrm{Bi}_{1.92} \mathrm{In}_{0.02} \mathrm{Te}_{2.85} \mathrm{Se}_{0.15}$.
\end{abstract}

